\title{
INSULIN RESISTANCE IN PREPUBERTAL CHILDREN IN RELATION TO INTRAUTERINE
} EXPOSURE TO OVERNUTRITION

\author{
O. Maftei $^{1,2}$, M.J. Whitrow ${ }^{1,2}$, V.M. Moore ${ }^{1,2}$, M.J. Davies ${ }^{1}$ \\ ${ }^{1}$ Research Centre for the Early Origins of Health and Disease, Robinson Institute, ${ }^{2}$ Discipline of Public \\ Health, University of Adelaide, Adelaide, SA, Australia
}

Background and aims: Intrauterine factors contributing to fetal overnutrition have been associated with greater insulin resistance in neonates, however it is unknown whether this persists into childhood.

We aimed to examine associations of maternal pre-pregnancy obesity, glucose intolerance during pregnancy and gestational weight gain with offspring insulin resistance before puberty.

Methods: We analysed data from a prospective birth cohort study $(\mathrm{n}=557)$ in Adelaide, Australia, recruited during 1998-2000. At 9.5 years, 443 children participated, 163 providing a fasting blood sample. The main exposures were:

(1) maternal pre-pregnancy BMI;

(2) glucose tolerance during pregnancy: normal glucose tolerance (NGT), borderline gestational glucose intolerance (BGGI) defined by positive oral glucose challenge test and negative oral glucose tolerance test, gestational diabetes (GD);

(3) gestational weight gain (GWG). Child insulin resistance was estimated by homeostasis model assessment (HOMA-IR). Generalised linear models with log link function and Gaussian family were applied to assess the associations.

Results: Exposure to GD at 9.5 years was associated with higher HOMA-IR relative to offspring of mothers with NGT, robust to confounder adjustment $(42.9 \%$, 95\%CI 20.9-68.9) and attenuated by current BMI zscore (mediator) adjustment $(39.8 \%, 95 \% \mathrm{CI} 21.9-60.4)$. Exposure to BGGI was associated with a reduction in HOMA-IR after controlling for current BMI z-score $(-17.9 \%, 95 \% \mathrm{CI}-29.9,-3.96)$. There was no association between maternal pre-pregnancy BMI or GWG and child HOMA-IR.

Conclusions: Gestational diabetes appears to have an independent effect on child insulin resistance, reinforcing the importance of screening for and management of abnormal glucose tolerance during pregnancy. 